\title{
Platon et la philosophie analytique
}

Deux exemples : la méthode diérétique et le " troisième homme "

\section{Dorothea Frede}

Traducteur : André Laks et Joëlle Masson

\section{OpenEdition}

\section{Journals}

Édition électronique

URL : http://journals.openedition.org/philosant/1070

DOI : 10.4000/philosant. 1070

ISSN : 2648-2789

\section{Éditeur}

Presses universitaires du Septentrion

\section{Édition imprimée}

Date de publication : 1 novembre 2011

Pagination : 127-149

ISBN : 978-2-7574-0356-3

ISSN : 1634-4561

Référence électronique

Dorothea Frede, «Platon et la philosophie analytique », Philosophie antique [En ligne], 11 | 2011, mis en ligne le 01 novembre 2018, consulté le 19 avril 2019. URL : http://journals.openedition.org/ philosant/1070 ; DOI : 10.4000/philosant.1070

La revue Philosophie antique est mise à disposition selon les termes de la Licence Creative Commons Attribution - Pas d'Utilisation Commerciale - Pas de Modification 4.0 International. 


\author{
PLATON ET LA PHILOSOPHIE ANALYTIQUE \\ Deux exemples : la méthode diérétique et le « troisième \\ homme» \\ Dorothea FREDE \\ Université de Californie à Berkeley
}

RESUME. Que la philosophie ancienne ait bénéficié de certains raffinements méthodologiques dus à la philosophie analytique n'est guère mis en question, même par ceux qui ne s'en réclament pas. À la grande époque de la philosophie analytique, certains de ses meilleurs représentants étaient encore fort versés en histoire de la philosophie et appliquaient leurs compétences analytiques à ce qu'ils considéraient comme des problèmes centraux chez les auteurs anciens. Cet article suggère à travers deux exemples que, s'agissant de Platon, cette attention n'a pas eu seulement des effets revigorants, mais également déformants. Dans le premier cas, la méthode diérétique si chérie de Platon a été marginalisée par d'éminents philosophes analytiques qui l'ont considérée comme triviale et inintéressante. Dans le second cas, le problème de l'«auto-prédication » des Idées, qui est celui de savoir si les Idées sont des entités qui possèdent les propriétés qu'elles représentent, a été considéré d'une importance décisive pour la théorie platonicienne des Idées. À cause de ses conséquences supposément désastreuses, mentionnées une unique fois dans le Parménide, on a pensé que Platon avait renoncé à cette théorie sous sa forme classique. Au cours des dernières années, l'importance de la méthode diérétique a été réévaluée et celle du problème de l'auto-prédication réduite à de plus justes proportions. Cela ne diminue pas la valeur des contributions des philosophes analytiques à l'étude de Platon, mais signifie seulement qu'elles doivent n'être acceptées qu'avec précaution.

SUMMARY. That ancient philosophy has been benefited from certain methodical refinements that are due to analytic philosophy is hardly questioned even by specialists who are not adherents. In the heyday of analytic philosophy some of its best practitioners were still well versed in history of philosophy and applied their analytic skills to what they regarded as central problems in ancient authors. This article suggests via two examples, that in Plato's case this attention had not only invigorating but also distorting effects. In the first case, the diairetic method, much cherished by Plato, was shunted aside by prominent analytic philosophers as trivial and uninteresting. In the second case, the problem of the 'self-predication' of the Forms, i.e. whether the Forms

Philosophie antique, $\mathrm{n}^{\circ} 11$ (2011), 127-149 
are entities that possess the properties they stand for, has been taken as critical for Plato's Theory of Forms. Because of its allegedly disastrous consequences, mentioned by Plato only once in his Parmenides, he has been taken to have given up that theory in its classical form. In recent years the importance of the diairetic method has been resurrected and that of the problem of self-predication been scaled down. This does not diminish the value of the contributions of analytic philosophers to the study of Plato. It just means that they should be received with due care. 


\section{Préliminaire : Philosophie et histoire de la philosophie}

Les années cinquante et soixante du $\mathrm{XX}^{\mathrm{c}}$ siècle furent à beaucoup de points de vue des années fastes non seulement pour la philosophie en général mais en particulier pour la philosophie antique. Cette affirmation ne fera pas l'unanimité, parce que je me réfère ici à la philosophie analytique, qui n'a bien évidemment pas partout que des partisans. Mais même si l'on ne tient pas cette orientation pour l'alpha et l'oméga, il est indéniable qu'elle a donné à la philosophie antique de nouvelles impulsions - et, avant tout, qu'elle a motivé des jeunes gens hautement doués à s'y consacrer en se dotant pour ce faire de l'outil nécessaire. Il n'est pas ici question d'entonner une louange aux bienfaits dont cet accroissement de technicité, en particulier dans le domaine de la logique et de la philosophie du langage, a fait bénéficier la philosophie antique. Mais il n'y a aucun doute que cette effervescence, qui commença par régner dans les pays anglo-saxons, a aussi envahi peu à peu d'autres espaces culturels - et que, même chez des spécialistes qui n'étaient pas des défenseurs ardents de la philosophie analytique, un nouvel esprit s'est fait jour. Ce nouvel esprit consistait tout simplement, d'un point de vue philosophique, à élever le niveau d'exigence quant à l'interprétation particulière des grands esprits de la tradition occidentale ${ }^{1}$ :

1. Cela peut sonner comme une manière simplificatrice d'enjoliver les choses. Car il serait décisif de se demander plus avant si l'on était parfaitement au clair sur les motifs de ce mouvement : ce que l'on cherchait était-il une nouvelle lecture de Platon pour prouver que ce philosophe, auprès duquel on avait dans une certaine mesure pris ses premières leçons de philosophie, était parfaitement à la hauteur des nouvelles exigences en matière de questions philosophiques ? Ou bien voulait-on, à l'inverse, offrir une nouvelle lecture de Platon pour légitimer les nouvelles recherches (Platon avait déjà vu ce que l'on venait soi-même de laborieusement élaborer) ? Ou encore s'agit-il de quelque laborieuse combinaison des deux 
le plus grand raffinement philosophique, au moins pour ce qui est de l'horizon du questionnement, était bien le moins que l'on pouvait offrir à nos héros.

Cette effervescence philosophique s'est depuis longtemps dissipée. Certes, la philosophie analytique, la philosophie du langage et de bonnes connaissances en logique font désormais aussi partie du bagage philosophique des historiens de la philosophie - mais l'on perçoit désormais qu'il s'agit là d'outils et non de la voie royale vers la vérité. Que cette conscience s'accompagne d'une certaine désorientation et d'un certain désarroi chez ceux des philosophes qui cherchent à justifier leur activité, on ne peut guère l'ignorer. Il est vrai qu'un tel désarroi ne règne pas chez les spécialistes de philosophie antique - car les œuvres des grands penseurs du passé continuent de susciter l'intérêt -, mais on ne peut ni soutenir que cette spécialité attire aujourd'hui les meilleurs esprits (à moins qu'ils ne disposent déjà d'un fort penchant pour l'histoire), ni que la philosophie analytique joue un rôle central dans les discussions actuelles, qu'il s'agisse des thèmes traités ou de la méthode. Au reste, l'éditeur de la revue Phronesis a dès 1990 mis le doigt sur ce point de manière saisissante à propos de l'interprétation de Platon : « Il existe des signes que nous avons atteint une nouvelle étape dans la transition : le paradigme lui-même [de l'analyse des analyses] est en train de s'émousser; le programme de recherche est entré dans une phase scolastique, et la recherche de paradigmes nouveaux est en cours ${ }^{2}$. »

Mon intention, cependant, n'est pas de réfléchir sur les « hauts » et les «bas » des études relatives à l'histoire de la philosophie. Il ne s'agit pas non plus pour moi de démontrer que l'histoire de la philosophie profite toujours de la qualité de la philosophie contemporaine - ou encore que cette dernière lui porte préjudice. Cette relation est archi-connue. Mon propos est tout autre. Si la période «faste» des années cinquante et soixante fut particulièrement fructueuse pour la philosophie antique, c'est parce que les philosophes éminents étaient aussi à l'époque éminemment compétents dans le domaine de la philosophie antique. Le grec, le latin et les langues les plus importantes de la culture européenne contemporaine appartenaient alors encore au patrimoine culturel commun. Qui plus est :

perspectives: ce qui nous préoccupe nous-mêmes beaucoup, on le trouve aussi chez les autres - parce que à certains égards, cela a toujours été là ?

2. «There are signs that we have now reached another stage of transition: the paradigm [sc. of analyzing analyses] itself is becoming frayed at the edges: the research programme has entered a scholastic phase; and the search for alternative paradigms is under way. 》 (Schofield 1990, p. 327.) Cf. infra, p. 140. 
l'étude de la philosophie antique faisait partie intégrante de la formation philosophique générale. En conséquence de quoi les représentants les plus distingués de la philosophie anglo-saxonne pouvaient avoir deux cordes à leur arc. Il suffira ici de citer quelques noms : ils vont de Gilbert Ryle, John Austin, David Ross, Elisabeth Anscombe, Peter Geach, William et Martha Kneale, W.v. O. Quine, Georg Henrik v. Wright, jusqu’à Wilfried Sellars, Bernard Williams et Donald Davidson. Tous ont donné la preuve qu'ils ne produisaient pas seulement des interprétations philosophiquement intéressantes, mais que celles-ci pouvaient aussi satisfaire les plus hautes exigences sur le plan philologique. Dans cette perspective, le nom de David Ross se détache tout particulièrement, lui qui, pour beaucoup d'entre nous, est moins connu comme philosophe que comme un éminent interprète d'Aristote et un grand philologue, mais dont l'œuvre, particulièrement dans le domaine de l'éthique, jouit aujourd'hui d'un fort regain d'intérêt chez les philosophes systématiques ${ }^{3}$.

La page est désormais radicalement tournée. La génération actuelle des quarante et cinquante ans ne possède en général plus cette connaissance au reste, elle ne se rend d'ailleurs même pas compte qu'elle a ainsi perdu un bien des plus précieux. Cela ne signifie naturellement pas que les spécialistes de philosophie antique ne s'occupent plus de philosophie; mais cela veut dire que l'inverse n'est presque plus jamais le cas, de sorte qu'en philosophie, l'histoire de la philosophie est souvent traitée comme un corps étranger - le plus souvent, il est vrai, avec respect, mais aussi avec une «bienveillante indifférence ». Cela a pour conséquence que beaucoup de jeunes étudiants doués ne prennent plus le chemin de l'histoire de la philosophie ou que, faute de connaître les langues, ils trouvent ce chemin impraticable. Parmi les candidats, on trouve de plus en plus de jeunes gens qui ont déployé de grands efforts pour apprendre un peu de grec à travers des cours intensifs, mais qui ne dominent pas le latin - pour ne rien dire des autres langues de culture contemporaines, à vrai dire indispensables pour participer à la discussion savante.

Il ne s'agit cependant pas de se lamenter sur la situation actuelle - peu favorable à la philosophie antique - et encore moins de glorifier de manière

3. On trouvera une série de références dans Skelton 2010, qui écrit à propos de The Right and The Good (Oxford, 1930): «Although Ross's view appeared to suffer at the hands of critics in the middle and late parts of the last century, recent interest in normative and metaethical intuitionism has sparked a renewed respect and admiration for his unique contribution to ethics. » 
tout à fait inappropriée « les années fastes ». Si chaque mouvement philosophique contemporain « fort » a des effets stimulants sur l'histoire de la philosophie, il connaît aussi ses scories. Et c'est justement ce point que je souhaite illustrer à travers les deux exemples annoncés dans le titre de ma contribution et qui sont tirés de la réception de Platon. Dans le premier cas, un procédé auquel Platon a attribué une grande valeur, surtout dans ses œuvres tardives, est récusé comme étant sans intérêt, voire sans pertinence. Dans le second cas, le problème de ce que l'on appelle l'« auto-prédication » des Idées, que Platon ne mentionne qu'une seule fois brièvement, est amplifié jusqu'à se présenter comme le problème-clé de la compréhension de sa philosophie.

\section{La méthode diérétique et son interprétation}

Parmi les procédures que Platon propose à titre de méthode d'investigation philosophique, peu ont trouvé aussi peu grâce aux yeux de la majorité des philosophes analytiques que le procédé dialectique fondé sur le rassemblement (synagoge) et la division (diairesis). Certes, le jugement de Gilbert Ryle, qui fut longtemps la norme, n'est pas aussi dévastateur qu'on l'a souvent répété; mais il est vrai qu'il n'accorde qu'un crédit des plus limités à cette méthode, dont la seule valeur à ses yeux est de constituer un exercice pour débutants. Voici ce qu'il écrit dans Plato's Progress ${ }^{4}$ à propos de la composition du Sophiste:

Le Sophiste consiste, bizarrement, en un déploiement hautement abstrait et sophistiqué de raisonnement philosophique, pris en sandwich entre quelques opérations de division, lesquelles ne présupposent pas la moindre sophistication philosophique. Dans l'argumentation philosophique, la dialectique, ici équivalente à la philosophie, est décrite en $253 \mathrm{c}$-d comme science qui découvre comment «les plus grands genres» sont à la fois « joints » et « disjoints ». Le terme « division » apparaît ici une ou deux fois au milieu de nombreuses autres métaphores. Il est tentant d'en inférer, bien que cela ne soit pas nécessaire, que Platon estimait que construire l'échelle des genres n'était pas seulement une tâche propédeutique au travail du philosophe ou du dialecticien, mais qu'elle en était une partie, voire se confondait avec elle. Mais nous devons alors reconnaître que l'exploration par l'Étranger des dépendances et indépendances mutuelles des «plus grands genres » ne produit pas la moindre échelle de genres, aussi courte soit-elle. Car les plus grands genres ne sont pas reliés l'un à l'autre comme des genres à des espèces ou comme des espèces à d'autres espèces...

4. Ryle 1966. On trouve une discussion critique de cet ouvrage dans Freis 1969. 
L'Étranger ne produit pas de divisions, qu'elles soient dichotomiques ou trichotomiques; il ne produit aucune division du tout ${ }^{5}$.

Plus loin dans ce livre, il souligne à nouveau le rôle purement propédeutique des diérèses : « les divisions qui nous apparaissent infiniment pénibles et philosophiquement infructueuses dans le Sophiste et dans le Politique pourraient s'expliquer et se justifier comme une contribution personnelle de Platon à cette partie préliminaire du curriculum. » D'où le jugement que le Sophiste est « un sandwich maladroitement composé dans lequel le pain pourrait n'avoir valeur éducative que pour les débutants et la viande n'avoir de valeur que pour les seuls jeunes dialecticiens hautement qualifiés ${ }^{6}$. 》

L'opinion selon laquelle il n'y a guère à tirer de ce procédé s'était déjà exprimée longtemps avant les invectives de Ryle. Ainsi F.M. Cornford, dans son commentaire sur le Sophiste de $1935^{7}$, a-t-il tout simplement omis la traduction des longues séries de diérèses, de la détermination du pêcheur à la ligne jusqu'à celle du sophiste comme « expulseur des opinions fausses » et leur a substitué une courte paraphrase. Tout en soulignant que Platon accordait une grande valeur à la méthode diérétique, il considère pouvoir renoncer à quatorze pages de l'édition Estienne et remarque à ce sujet :

L'espèce doit être définie par division systématique du genre qui est supposé l'inclure. La méthode était nouvelle pour le public de Platon ; mais le lecteur moderne, familier des classifications qui dérivent toutes en fin de compte du modèle établi ici, pourrait être lassé par une traduction. C'est pourquoi je ne donnerai qu'un résumé des divisions, afin d'illustrer le propos... $^{8}$

En bref, les diérèses, d'un point de vue philosophique, sont une vieillerie. Il se trouve que le commentaire de Cornford précède la grande époque de la philosophie analytique. Cornford partage cependant un présupposé décisif avec ses collègues analytiques, à savoir la conviction que la logique moderne a transformé la philosophie de fond en comble. Ainsi, il explique de manière condescendante, s'agissant du fait que Platon ne connaissait encore rien des relations, et parlait à la place de propriétés relatives

5. Ryle 1966, p. $139 s q$.

6. Ryle 1966, p. 285 sq.

7. Cornford 1935.

8. Cornford 1935, p. 170. 
(comme «être le père de... »): «il était réservé à des logiciens encore vivants de découvrir qu'une proposition telle que "Socrate est plus petit que Phédon" comporte deux sujets unis par une relation et pas le moindre prédicat ${ }^{9}$. » Avec des nouveautés de ce type, ni les arborescences de genres et d'espèces, si familières depuis Linné à tous ceux qui s'intéressent à la biologie, ni leurs antécédents antiques, ne sauraient naturellement entrer en concurrence.

Les attentes et évaluations auxquelles Ryle soumet la philosophie de Platon sont du même type que celles de Cornford. Si en effet on se demande pourquoi ce dernier estime tellement important l'entrelacement mutuel des plus grands genres (à savoir Être, Identité, Différence, etc.) la réponse est sans équivoque :

On peut soutenir que dans le Sophiste même, Platon commence à explorer à tâtons ce que nous pouvons identifier - ce que lui n'était pas encore en position de faire - avec les implications, les incompatibilités et les compatibilités existant entre les propositions en vertu des concepts « communs » qu'elles renferment. [...] S'il en est ainsi, [...] alors dans le Sophiste [...] il assigne au dialecticien un type de recherche que nous appelons maintenant recherches logiques ${ }^{10}$.

Pour l'exprimer de manière tout à fait terre-à-terre, ce qui se manifeste ici est la conviction que la philosophie n'a pas à nous présenter des contenus nouveaux, mais qu'elle a pour tâche de mettre à jour des structures logicosémantiques.

Mais sur quoi tout cela débouche-t-il ? Pour ce qui est du Plato's Progress, Ryle considère que la chose la plus importante est la réfutation (l'elenchos), et ce justement non pas en tant que moyen en vue de trouver la vérité ou de bannir l'erreur, mais en tant que jeu dialectique, tel qu'on s'y adonnait certainement dès l'époque de Socrate et tel que les sophistes l'enseignaient. L' « élenchtique », ou art de la réfutation, est un événement sportif intellectuel où il ne s'agit que de mettre son adversaire échec et mat au moyen d'un questionnement habile, et si possible sur la base de questions auxquelles on puisse répondre par « oui » ou par «non », selon la procédure décrite par Aristote dans les Topiques.

Il n'est pas nécessaire de réfléchir bien longtemps pour voir que cette démarche ne rend pas justice à la nature du questionnement socratique. Les

9. Cornford 1935, p. 284.

10. Ryle 1966, p. 142. 
questions auxquelles on peut répondre par « oui » ou par «non»sont rares - et Socrate ne se présente pas plus en position de compétiteur qu'il ne se retire en vainqueur : sa fonction est bien plutôt celle d'un questionneur s'efforçant d'appréhender une vérité qui la plupart du temps ne se laisse malheureusement pas découvrir. S'il s'était véritablement agi pour Socrate d'user d'arguments « imparables », il devrait être considéré comme l'un des plus grands hypocrites de toute l'histoire, puisqu'il se présente toujours comme soucieux de la vérité et de l'âme de ses interlocuteurs.

Je ne parlerai pas ici des aspects les plus aventureux du Platon de Ryle, notamment de l'idée que c'est à la suite d'une décision judiciaire que Platon aurait été contraint à abandonner l'éristique. Ce qui est intéressant ici est entre autres que Ryle fasse de Platon un philosophe quelque peu lent à réagir. Car si la décision judiciaire en question date de la toute fin des années 370, et que ce n'est qu'alors qu'il a remarqué que la réfutation est une chose et que la résolution d'un problème philosophique en est une autre, on peut soutenir, pour parler communément, qu'il est longtemps « resté dans le brouillard », puisqu'il avait à l'époque déjà presque soixante ans. C'est alors seulement qu'il se serait concentré sur les Idées et les problèmes philosophiques que celles-ci soulèvent. Dans cette vision des choses, Platon doit avoir vraiment beaucoup travaillé par la suite - puisqu'il a dû écrire toutes les œuvres qui s'écartent de la pratique éristique après 370 , toutes, y compris l'Apologie, le Phédon, la République - et naturellement aussi les dialogues tardifs qui à nouveau s'éloignent des Idées. Comment il a pu accomplir tout cela dans les vingt dernières années de sa vie relève du miracle. Mais nous en resterons là avec le Platon de Ryle, puisque aussi bien il est aujourd'hui généralement traité comme une curiosité.

Naturellement, tous les philosophes de tendance analytique n'ont pas voulu restreindre le vrai Platon à la seule dimension du logico-formel, et avec la renaissance de la métaphysique au sein de la philosophie analytique, une page a de toute façon été tournée, c'est-à-dire que l'on prête à nouveau depuis longtemps attention, chez Platon, aux contenus effectifs et pas seulement à la forme logique. Pourtant, un auteur aussi important que Donald Davidson pouvait affirmer en 1990, dans une préface écrite à l'occasion de la publication de sa dissertation sur le Philèbe (qui date de 1949 et avait été écrite sous la direction de Werner Jaeger), que le Philèbe était le signe d'un retour de Platon à l'essentiel : à l'elenchos. Quiconque connaît le Philèbe trouvera cette affirmation aussi étonnante que problématique. Car le dialogue ne contient qu'un unique mini-elenchos, celui par lequel Socrate dé- 
montre à Protarque qu'une vie dépourvue de toute connaissance équivaut à la vie d'un mollusque ou d'un coquillage marin ${ }^{11}$. On ne trouve rien de plus dans le dialogue en matière de réfutation - au reste, Davidson n'avait originellement affirmé rien de tel dans sa dissertation.

Le fait que l'on ait pu s'obstiner de la sorte à propos de ce que pouvait être ou non l'intention de Platon a naturellement son fondement dans la métaphysique platonicienne. Car la méthode diérétique ne peut être appliquée que dans les seuls cas où nous nous trouvons devant un genre complet, avec ses sous-genres, ses espèces et sous-espèces. Cela vaut par exemple pour les vertus, pour les lettres, pour les humains et les animaux et pour les disciplines artisanales. Car une fois que l'on a intégralement établi au sein de tel ou tel genre un ordre taxinomique, on a atteint l'objectif dont Platon fait son point de départ dans plusieurs de ses dialogues postérieurs: parvenir à distinguer un phénomène de tous les autres phénomènes similaires, c'est suffisamment cerner la nature de la chose en question. Or il y a beaucoup à dire en faveur de l'hypothèse selon laquelle Platon a, après le Ménon et le Phédon, abandonné la théorie de la réminiscence au profit de la collectio et de la divisio. En témoigne en particulier un passage central du Phèdre. Au milieu du tableau mythique qu'il dresse du destin des âmes les meilleures et des moins bonnes, Platon explique sans hésiter que seules ont accès à la vérité les âmes de ceux qui sont capables de saisir un ensemble « selon l'eidos » et, partant de nombreuses perceptions, de rassembler les phénomènes sous un concept $(249 b-c)$. Platon insère donc ici, au milieu du tableau mythique de l'ascension de l'âme vers le lieu supra-céleste des Idées, une remarque méthodologique préliminaire qu'il décrira, dans le cours ultérieur du dialogue, comme la méthode de rassemblement sous un genre et de division en espèces et qu'il caractérisera comme « art divin de la dialectique » (265d-266c). Pourquoi Platon fait-il si grand cas de cette méthode, au point même de la décrire dans le Philébe comme « cadeau des dieux aux hommes, à l'aide duquel nous cherchons, apprenons, et nous enseignons les uns aux autres » (16c5-e4) ? La réponse est fort simple : cette méthode lui permet de trouver de façon systématique des réponses à ses questions de type «qu'est-ce que $\mathrm{X}$ ? ». Car une fois qu'on a fixé le domaine auquel appartient un objet déterminé, et qu'on a différencié celui-ci de tous les phénomènes apparentés, le résultat finalement obtenu est la définition de l'objet en question. 
Que de nombreux philosophes, en particulier analytiques, ne partagent pas cette appréciation, et ne consentent à accorder aux diérèses qu'une valeur tout au plus propédeutique, tient à de nombreuses raisons. Premièrement, même le plus chaud sympathisant devra bien admettre que l'application que Platon fait de la méthode laisse beaucoup à désirer. Il suffit de penser aux nombreuses diérèses à l'aide desquelles le sophiste doit être défini (Sophiste, 219a-231b) - ou pire encore, aux longues séries de diérèses qui nous sont imposées dans la recherche de l'«homme politique » (Politique, 258b-267c;279b-283b; 287d-290b) - pour que la majorité d'entre nous abandonne tout enthousiasme pour cette méthode. Car les subdivisions sortent tout simplement du chapeau sans la moindre justification, comme si elles allaient entièrement de soi. Naturellement, une question est de savoir pourquoi Platon se montre si peu scrupuleux dans l'exécution effective de ses diérèses; tout autre est celle de savoir s'il pensait satisfaire ainsi ses propres exigences. Or que tel soit le cas, on peut le récuser à bon droit. Platon explique en effet clairement à plusieurs reprises que les diérèses doivent être complètes, exécutées dans l'ordre correct (Philèbe, $16 c-17 a$ ) selon des critères adéquats (« découpées selon les articulations », Phèdre, 265e-266a), et éviter les erreurs dues à des phénomènes linguistiques (Politique, 262b-263c). Platon n'a sans doute pas jugé approprié d'exécuter concrètement ce programme «dans les règles de l'art », ne serait-ce que dans une certaine mesure, car pour cela, il aurait été besoin de considérations et de déterminations si minutieuses (s'agissant par exemple de la bonne différence spécifique) qu'elles auraient fait exploser la forme du dialogue. Il est vrai que ce choix a nui à la chose même, comme le montre le fait que la méthode n'a guère été l'objet d'appréciations positives.

Bien entendu, tous les philosophes ne se sont pas rangés au jugement de Ryle sur la méthode diérétique. John Ackrill, en particulier, s'est très tôt opposé à lui de façon énergique. Dans son article « Pour la défense de la division platonicienne », il a montré qu'il ne saurait être question que Platon pratique cette méthode purement et simplement à titre d' « exercice introductif» destiné aux philosophes de première année ${ }^{12}$. L'explication d'Ackrill insiste sur les points suivants : (1) même s'il est vrai que l'enthousiasme de Platon pour la méthode diérétique n'est pas exempt d'exagération, elle constitue néanmoins un auxiliaire utile à la découverte des définitions, et donc pour apporter une réponse aux questions de type 
«qu'est-ce que X ? »; (2) les diérèses ne peuvent pas être établies par voie déductive, car la structure d'un genre avec ses différentes espèces n'est rien que l'on puisse connaître ou déduire a priori. Que les recherches empiriques interviennent également dans sa constitution ne signifie pourtant pas que l'ordre interne à un genos ne soit pas objet de réflexion philosophique. Il n'existe donc aucune ligne de démarcation nette entre les questions empiriques et les questions conceptuelles - ce qu'on pourait assez joliment montrer sur l'exemple de la découverte de la classification des lettres par Theuth, le dieu égyptien de l'écriture, dont Platon parle dans le Philèbe (18b5-d2) : naturellement, on ne peut déduire quel type de lettres existe - il faut déjà les connaître et en dresser un inventaire pour être certain de l'ordre sur lequel ce domaine repose. La classification adéquate des éléments d'un domaine déterminé n'en est pas moins objet de réflexion philosophique.

En outre, la recherche d'une répartition en genres et en espèces conduit aussi à découvrir d'importantes ambiguïtés, dont Aristote fera volontiers son profit : comme Ackrill le montre de manière fort plausible, la méthode diérétique de Platon a fortement contribué à aiguiser la sensibilité aux équivoques conceptuelles et à leurs causes. Plus important encore: Ackrill défend l'idée que l'on ne devrait pas lier les diérèses de Platon aux nomenclatures à la Linné. Celles-ci sont certes les descendantes de la méthode platonicienne, mais le procédé n'est aucunement limité chez Platon aux genres et aux espèces biologiques. La diairesis peut bien plutôt s'appliquer à des domaines très variés et conduit à des différenciations de toute sorte.

Ackrill ne conteste pas que l'usage que fait Platon de la méthode diérétique, en particulier dans le Sophiste, ne la recommande guère à titre de méthode philosophique. Il pense cependant qu'une interprétation suffisamment généreuse de ce que Platon entreprend dans le Sophiste rend superflue l'opposition, que Ryle y projette, entre les diérèses, qui seraient un accessoire inutile, et « les genres les plus importants » (les megista gene), dont la tâche propre du philosophe serait de s'occuper. Ackrill soutient là contre, et à juste titre, que l'on ne devrait pas réduire le passage central du Sophiste où Platon explique ce qu'est la tâche propre du philosophe (253be) au rapport de compatibilité et d'incompatibilité des concepts formels tels que l'Être, l'Identité et la Différence, mais que Platon intègre également à l'entrelacement des plus grands genres (megista gene) les relations entre les Idées générales existant entre genres et espèces. Aux yeux d'Ackrill, ceci ne 
représente pas une extension illégitime de l'«entrelacement des Idées » (symploke eidon); il soutient au contraire que tel est précisément l'objectif de Platon dans le Sophiste. Il n'existe donc pas pour Platon de séparation rigoureuse entre l'application de la méthode diérétique et la mise en évidence de la compatibilité et de l'incompatibilité des plus grands genres : on doit bien plutôt supposer un rapport de complémentarité. De fait, beaucoup de choses dans le Sophiste vont en ce sens. Car l'opposition tranchée entre l'étude du rapport qu'entretiennent les concepts formels généraux, d'une part, et les concepts classificatoires, d'autre part, que Ryle impute à Platon, ne se laisse pas repérer dans le dialogue. Les deux tâches relèvent apparemment du domaine du dialecticien.

Vu la position critique d'Ackrill face aux réserves formulées par certains philosophes analytiques à l'encontre de la doctrine des Idées, on ne peut, il est vrai, qu'être surpris de ce que, dans un autre passage, il se rallie à la position des «analytiques » en vertu de laquelle la critique des Idées dans le Parménide permet de conclure que Platon a totalement abandonné les Idées telles qu'elles se présentent dans ses dialogues intermédiaires :

Quoi qu'il en soit, le passage cité [à savoir Parménide, 135b) suggère fortement que ce dont il (scil. Platon) est désormais sûr, ce n'est pas qu'il doit exister des formes du type de celles qui apparaissent dans les dialogues intermédiaires, à savoir en tant qu'idéaux éthiques et objets métaphysiques accessibles à une connaissance intuitive et peut-être mythique; ce dont il est maintenant sûr, c'est qu'il doit exister des objets fixes pour garantir le sens du discours, des concepts fixes - la signification des termes généraux dont le rôle est d'assurer ten tou dialegesthai dynamin [la capacité de s'entretenir $]^{13}$.

Que Ackrill fût prêt à se rallier à ce sujet aux interprètes « révisionnistes » doit certainement aussi être imputé à l'« esprit du temps », dont le présupposé était que le renoncement à la conception des Idées paradigmatiques dans sa forme originelle était «mûr » et que celles-ci devaient être remplacées par les megista gene du Sophiste. Avec des concepts aussi généraux qu'Être, Identité, Différence, Unité, Pluralité, Ressemblance et Dissemblance, on n'a certainement pas les mêmes problèmes qu'avec les Idées de Vertu, de Beau et de Bien. Il n'est pourtant aucunement certain que Platon ait vraiment voulu se débarrasser des Idées de ce genre - et ce, pas même dans le Sophiste. En effet, après avoir parlé des megista gene et de 
leur rapport mutuel, il associe sans la moindre médiation, dans la discussion ultérieure du rapport entre les choses qui sont (onta) et celles qui ne sont pas (me/ouk onta), de vieilles connaissances: le Grand, le Petit et l'Égal figurent ici tout comme le Beau et le non-Beau, le Juste et le non-Juste $(257 b-258 b)^{14}$. Cela ne semble pas avoir été pris en compte par les interprètes révisionnistes, quand ils pensent que dans le Sophiste, Platon a remplacé les Idées de vieille facture par les megista gene. En réalité, il semble maintenir à la fois les unes et les autres.

\section{L'argument du troisième homme}

Alors que la querelle relative aux diérèses n'a constitué qu'un théâtre de guerre secondaire, l'«autocritique » que Platon adresse à la doctrine des Idées dans le Parménide s'est trouvée durant plusieurs décennies au centre des débats. Car même les « amis des Idées » ne peuvent éviter d'admettre que les questions que Platon formule dans ce dialogue sont autant de difficultés pour un partisan des Idées et que, loin d'être résolues au cours de l'entretien, elles demeurent sans réponse. Une fois réfutées toutes les tentatives du jeune Socrate pour justifier les Idées, Parménide se contente de constater qu'abandonner les Idées signifierait renoncer à une pensée claire et à l'explication philosophique (135b5-c2). Certes, il n'indique pas à quoi devrait ressembler la solution des difficultés, et on discute jusqu'à aujourd'hui pour savoir si les «exercices dialectiques » de la deuxième partie du dialogue offrent ne serait-ce qu'un fondement à une telle solution. C'est la raison pour laquelle une lutte opiniâtre a éclaté, parmi les interprètes, entre « unitariens », « révisionnistes » et « évolutionnistes » autour de la signification de cette autocritique - lutte qui n'a pas été véritablement tranchée jusqu'à aujourd'hui. Il serait plus juste de dire que, au vu de la récurrence ininterrompue d'arguments pro et contra, une certaine lassitude s'est installée, qui est reflétée dans le diagnostic de Malcolm Schofield relatif au caractère scolastique de ce débat ${ }^{15}$. Ces dernières années, en tout cas, le flot des publications relatives au «troisième homme » a fortement diminué.

Deux raisons plaident contre l'idée que la critique des Idées, dans la première partie du Parménide, constitue une ligne de partage dans la pensée

14. La même chose vaut pour le catalogue des « concepts communs » (koina) dans le Théétète. Il comprend, outre l'être et le non-être, la ressemblance et la dissemblance, l'identité et la différence, l'unité et la pluralité, le beau et le laid, le bon et le mauvais (185c-186c).

15. Voir supra, p. 130. 
philosophique de Platon. La première tient aux moyens dramatiques mis en œuvre par Platon, la seconde au contenu même de la critique. Le dossier étant bien connu, un bref résumé suffira.

(1) Par moyens dramatiques, on doit entendre le fait que, dans une certaine mesure, la critique des Idées est située « dans la nuit des temps » : un Socrate encore très jeune avance une doctrine des Idées à titre de contreargument au paradoxe de Zénon relatif à la pluralité. Son idée est qu'il n'y a rien d'étonnant à ce que les objets qui nous sont familiers soient à la fois uns et multiples et plus généralement contradictoires, mais qu'il serait très étrange que de telles contradictions puissent être mises en évidence dans le cas des Idées elles-mêmes. Le fait que ce très jeune Socrate, impertinent comme il est, ne soit pas à la hauteur de la critique de Parménide, ne prouve encore rien du tout en soi. Cela montre simplement que les tenants d'une telle théorie doivent s'attendre à répondre à des questions critiques. Que le tout jeune Socrate ne se montre pas à la hauteur de ces questions ne signifie pas que Platon ne l'ait pas été - cela plaide plutôt en faveur du contraire. Si Platon confie à un très jeune Socrate d'exposer une théorie des Idées et de la défendre contre Parménide, il a pu vouloir présenter une version de la doctrine qui requiert encore élaboration et justification. Cela ne prouve pas qu'il ait lui-même jamais défendu une théorie aussi fragile. Les nombreux renvois à la grande jeunesse de Socrate et à son défaut d'exercice parlent plutôt là contre, d'autant plus qu'on ne trouve encore aucune théorie des Idées dans les dialogues de Platon dits « de jeunesse ».

(2) La nature même des critiques de Parménide est, notoirement, de qualité très variable, au point qu'on s'étonne que même le jeune Socrate les accepte toutes sans contradiction. Cela vaut en particulier pour le premier des cinq arguments ${ }^{16}$, c'est-à-dire pour la question de savoir si les choses qui participent à une Idée y participent en totalité ou simplement pour une partie. Ni l'une ni l'autre hypothèse ne s'avère possible : si l'on prend à la lettre le terme de «participation », chaque chose devrait ou bien « posséder » l'Idée entière, de sorte que l'Idée dans son ensemble se trouve dans les choses qui y participent, ou bien n'en avoir qu'une partie - et une chose serait alors par exemple grande en vertu de ce qu'elle possède une petite partie de la Grandeur. Point n'est besoin d'un long développement pour expliquer pourquoi il est invraisemblable que Platon ait jamais pensé à la «participation » en termes de «possession d'une partie», comme s'il 
s'agissait d'un morceau de gâteau. Dans le Phédon, il se montre explicitement réservé quant à la dénomination précise de la «participation », puisque les termes de «présence » (parousia) et de «communauté » (koinonia) sont mentionnés comme des options possibles à côté de methexis («participation »). Il est vrai que Platon n'a jamais non plus explicité en quel sens il faut comprendre le terme de «participation », ce qu'Aristote lui a vivement reproché ${ }^{17}$. Si l'argument de la «participation » dans le Parménide a trouvé peu de défenseurs sérieux parmi les critiques de Platon, c'est sans doute que les Idées ne sont pas des choses possédant des « parts » au sens littéral du terme, même si cette réponse n'est pas venue à l'esprit du tout jeune Socrate, en proie à la confusion.

Tout différent est le cas de l'argument qui suit immédiatement, que l'on appelle l'argument $\mathrm{du}$ «troisième homme ». Cette appellation ne figure pas chez Platon, qui parle $\mathrm{du}$ «Grand », mais comme Aristote a traité de ce problème sous ce nom ${ }^{18}$, l'habitude a été prise de s'y référer ainsi. L'argument n'est pas seulement très connu; ce qui le rend si attrayant est manifestement sa brièveté : avec ses quatorze lignes (132a1-b2), c'est le plus court de tous ceux qu'avance Parménide contre la recevabilité des Idées. Depuis que Gregory Vlastos a soumis cet argument à une analyse détaillée, il n'a cessé d'être objet de critiques, d'améliorations et de clarifications ${ }^{19}$. Car il débouche sur le résultat paradoxal que l'Idée du Grand grâce à laquelle tout ce qui est grand est grand, et qui donc garantit l'« unité » des objets concernés, conduit pour sa part à une pluralité d'Idées, parce que l'Idée du Grand, en tant qu'elle est quelque chose de grand, exige à son tour une autre Idée du Grand qu'elle doit partager avec tout autre grand. Ainsi se produit une régression à l'infini des Idées, parce qu'à chaque nouvelle étape il faut poser une Idée supplémentaire qui soit commune à tout grand, y compris, à chaque fois, à la nouvelle Idée du Grand.

Cette reductio ad absurdum est fâcheuse, car au lieu d'une unique Idée du Grand en vertu de laquelle tout grand est grand, on obtient une série infinie d'Idées, sans que l'on puisse dire d'aucune qu'elle serait l'Idée en vertu de laquelle toutes les grandes choses sont grandes.

17. Metaph. A, 9, 991a19-23; An. Post. I, 22, 83a32-35.

18. Metaph. A, 9, 990b17; Z, 13, 1039a2-3.

19. Bref panorama bibliographique sur la question : Vlastos 1954; Sellars 1955; Vlastos 1955; Geach 1956; Sellars 1967; Cohen 1971; Teloh \& Louzecky 1972; Vlastos 1973; Hägler 1983; Quine 1987; Mignucci 1990 ; Meinwald 1992. La monographie récente de Lienemann 2010 offre un traitement détaillé de la problématique et de la littérature secondaire. 
Ce que Vlastos a fait est de relever les deux facteurs responsables de cette régression :

(1) ce qu'il appelle l'auto-prédication (AP) : l'Idée doit elle-même posséder la propriété qu'elle représente ;

(2) ce qu'il appelle non-identité (NI) : l'Idée n'est pas identique à la propriété qu'elle représente.

Mais à y regarder de plus près, il s'avère que ces deux suppositions ne s'accordent pas entre elles, c'est-à-dire que AP et NI ne sauraient être toutes deux vraies en même temps. Car s'il est vrai de l'Idée du Grand qu'elle est grande, on ne comprend pas la nécessité d'un autre principe, c'est-à-dire d'une Idée supplémentaire et différente d'elle, qui expliquerait qu'elle soit grande. Le regressus surgit seulement parce que l'on suppose que l'Idée du Grand possède elle-même une grandeur qu'elle aurait en commun avec toutes les autres choses grandes, de sorte qu'il semble qu'on ait besoin d'un standard supplémentaire pouvant expliquer en quel sens toutes les choses grandes sont grandes, y compris l'Idée même du Grand.

Les analogies pouvant servir à illustrer ce point ne manquent pas. Ainsi Peter Geach a-t-il par exemple renvoyé au mètre étalon de Paris, dont il serait insensé de prétendre qu'on a encore besoin d'un autre mètre étalon pour expliquer de quelle manière toutes les choses qui sont longues d'un mètre, y compris le mètre étalon lui-même, sont longues d'un mètre. Pour le dire en un mot : le mètre étalon est un mètre, mais sa longueur n'est pas à son tour celle d'un autre mètre.

Cette distinction n'apparaît pas immédiatement chez Platon, quand il présuppose que toutes les choses sont des objets de même ordre, qui tous possèdent la propriété du Grand au même sens du terme.

Je suppose que la raison qui te fait penser que chaque forme (eidos) est une est la suivante : quand plusieurs choses te semblent être grandes, sans doute estimes-tu, à toutes les considérer, qu'il y a une même Idée (idea), ce dont tu conclus que le Grand est un ${ }^{20}$.

Jusque-là, rien que d'anodin. Le pas décisif vient ensuite, avec l'affirmation que c'est la totalité des grandes choses qui doit être prise en compte: 
Et pour ce qui est du Grand lui-même et des autres choses grandes, si tu les considères toutes de la même manière avec l'âme, n'apparaîtra-t-il pas à son tour quelque Grand grâce auquel toutes ces choses apparaissent grandes ${ }^{21}$ ?

Cette prise en compte de toute chose grande, y compris de l'Idée du Grand, pouvant être répétée ad libitum, une régression à l'infini des Idées paraît finalement être inévitable.

Cette argumentation de Platon est déjà problématique à cause de son manque de rigueur. Pourquoi devrait-on, « avec l'âme », regarder « de la même manière » toutes les choses grandes et l'Idée du Grand ? N'est-ce pas le nœud de toute l'affaire, que de placer les Idées sur le même plan que les choses qui y participent ? Comme les Idées sont ce que les choses qui y participent ne font que représenter, une telle égalité de traitement semble tout à fait inadéquate. En refusant cette égalité de traitement, on bloque d'emblée la voie à une régression infinie. Naturellement, une telle solution satisfera tous ceux qui pensent que Platon ne voit dans les arguments aucune difficulté sérieuse, et qu'il veut seulement démontrer qu'une doctrine des Idées non réfléchie, comme l'est celle du jeune Socrate, s'expose à une manœuvre telle que celle de Parménide, quand il traite de manière indifférenciée les Idées et les choses qui y participent ${ }^{22}$.

L'autre parti, qui pense que, au regard de ces difficultés, Platon s'est senti obligé d'abandonner la doctrine des Idées de ses dialogues intermédiaires, voit évidemment les choses d'une tout autre manière. Ses adhérents mobilisent une artillerie argumentative lourde pour montrer que la régression à l'infini est valide. Les deux prémisses implicites inconciliables, c'est-àdire l'auto-prédication et la non-identité, sont alors soumises à des interprétations variées ${ }^{23}$. Naturellement, ce travail d'orfèvre n'est rentable que si l'on est effectivement d'avis que, avec l'âge, Platon n'a plus défendu d'Idées paradigmatiques, et que son point de départ ne lui est désormais plus fourni que par des concepts tels que les megista gene, qui ne créent pas tant d'embarras. Telle fut de fait l'interprétation dominante du temps de l'apogée de la philosophie analytique, et les voix dissidentes sont la plupart du temps restées fort discrètes sur la question. Le tableau s'est modifié au cours des deux dernières décennies. Ainsi, par exemple, Constance Meinwald a ex-

21. Parm. $132 a 6-8$.

22. Un Socrate plus mature aurait justement rejeté ce « considérer de la même manière avec l'âme ». C'est précisément ce que l'on ne fera pas, si l'on a compris la différence entre les Idées intelligibles et immuables et leurs participants matériels et changeants.

23. Et infiniment trop nombreuses pour être évoquées ici. 
pliqué dans son livre sur le Parménide que les paradoxes énoncés dans la première partie du dialogue sont simplement trop sous-déterminés pour autoriser un jugement sur la façon dont Platon lui-même les juge ${ }^{24}$. Un autre argument est que Socrate n'oppose aucune résistance et n'exprime pas le moindre doute à propos des apories, contrairement à ce qui se produit usuellement dans les premiers dialogues aporétiques de Platon. Mieux vaudrait donc prendre au sérieux les indications données par Platon luimême dans le cours du dialogue : à savoir, premièrement, qu'on a encore besoin des Idées si des discours signifiants doivent pouvoir exister; et en second lieu, qu'il faut se soumettre à une gymnastique intellectuelle laborieuse pour mieux comprendre quelles déterminations valent pour les Idées et quelles sont celles pour lesquelles ce n'est pas le cas. La première affirmation de Meinwald a trouvé un écho très favorable. Son analyse particulière des exercices dans la seconde partie du dialogue est moins heureuse : un examen plus approfondi révèle qu'il ne suffit pas de différencier comme elle le fait entre les déterminations qui conviennent à l'Idée considérée en elle-même (pros heauto) et celles qui conviennent à l'Idée considérée en rapport avec autre chose (pros allo) pour résoudre toutes les contradictions ${ }^{25}$.

La possibilité de trouver au problème du «troisième homme»des solutions permettant à Platon de maintenir la doctrine des Idées n'enlève pas toute valeur au plaidoyer des « analytiques » en faveur d'une position « révisionniste ». C'est incontestablement à l'interprétation analytique que revient le mérite d'avoir mis fin à un état de paralysie qui soustrayait la philosophie de Platon, chez beaucoup d'interprètes, à tout questionnement critique, en montrant que Platon lui-même soumet sa théorie à une critique sérieuse, et pour quelle raison. Car quelle que soit la façon dont on répond en fin de compte à la question de la nature des Idées, il est besoin d'expliquer ce que sont les Idées elle-mêmes, comment elles incarnent ce qu'elles entendent représenter, et la relation que les choses qui en participent entretiennent avec elles. Affirmer qu'il en va simplement ainsi chez Platon et que la nature de toutes les choses consiste pour lui en une participation des Idées ne constitue qu'un résumé purement formel de la problématique. Il ne fait aucun doute que la première partie du Parménide indique clairement que Platon lui-même discerne parfaitement les insuffisances liées à la sous-détermination de sa théorie, en particulier concernant

24. Meinwald 1991, en particulier Chap. 1, p. 5-19; voir également Meinwald 1992. 1998.

25. Pour une critique brève mais pertinente de la conception de Meinwald, voir Krohs 
le rapport des participants des Idées, mais aussi pour ce qui touche à la question du statut des Idées et de leur nature elle-même.

Il faut en revanche, de manière critique, porter au débit de l'approche analytique qu'elle a durant des décennies fait prévaloir le dogme que Platon ne fait pas que problématiser les insuffisances de la doctrine des Idées dans les dialogues intermédiaires, mais qu'il entendait s'en débarrasser entièrement - comme l'ont fait si longtemps ceux des spécialistes qui défendent une position révisionniste, et comme le font encore quelques irréductibles. Car ce dogme a alimenté beaucoup de discussions inutiles, avant tout concernant la question de la datation des dialogues. Puisque les Idées paradigmatiques devaient ne plus intervenir chez le Platon tardif, on était en effet contraint de réviser la chronologie : le Timée, avant tout, devait être considéré comme une œuvre appartenant à l'époque intermédiaire ${ }^{26}$. Les raisons qui parlent contre une telle révision de la chronologie ne peuvent être explicitées ici. Rappelons seulement encore le fait, déjà mentionné plus haut, que la « liste » des idées générales dans le Sophiste comporte aussi, à côtés des « concepts communs », les Idées paradigmatiques, et qu'il en va de même pour la liste figurant au début du Philébe (15a).

En eux-mêmes, ces efforts de révision ne doivent évidemment pas être jugés de façon purement négative : le niveau de discussion de la philosophie du Platon tardif s'en est trouvé relevé de manière décisive. S'il n'y a aujourd'hui pratiquement plus de taches blanches sur la carte géographique des œuvres de Platon, c'est-à-dire d'endroits où personne ne se risque ou dont personne ne reconnaît qu'ils lui sont inintelligibles, cela aura été le mérite essentiel des efforts des philosophes analytiques, pour avoir appliqué à Platon aussi toute la subtilité qui appartient depuis au patrimoine commun des philosophes. Les partialités et exagérations sont de toutes les époques de la philosophie. Les générations ultérieures les corrigent ensuite pour en produire pour leur part de nouvelles. C'est de cela que vit la philosophie et c'est un signe de la grandeur de Platon que de toujours aussi continuer à offrir un nouvel aliment aux partialités et exagérations philosophiques. Les grands philosophes sont en cela semblables aux grands artistes - et peutêtre pas seulement en cela.

En guise de confirmation et de divertissement final, j'évoquerai la critique que le très jeune Richard Sorabji adressait à l'époque à la manière

26. Renvoyons seulement au célèbre débat Owen/Cherniss concernant la place du Timée au sein des œuvres de Platon: cf. Allen 1965, p. 313-338 (Owen) et 339-378 (Cherniss). Comme exemple d'irréductible, on peut renvoyer à Bostock 1994. 
dont la philosophie analytique abordait Aristote ${ }^{27}$. Sorabji ne condamne aucune erreur d'interprétation, mais justifie la préférence évidente des philosophes d'Oxford pour l'Éthique à Nicomaque par le fait que cette œuvre est exempte d'un certains nombre de traits qui, pour des raisons philosophiques, ne plaisent guère à la corporation d'Oxford, ou ne les présente que de façon tout à fait marginale. Tous ne peuvent être mentionnés ici. Au nombre des caractéristiques de la philosophie aristotélicienne peu appréciées se trouve, selon Sorabji, la prédilection d'Aristote dans ses œuvres scientifiques pour des définitions obscures et des informations énigmatiques ${ }^{28}$. C'est pourquoi, dans ces œuvres scientifiques, les définitions sont à comprendre, en suivant la distinction de Locke, comme des définitions réelles plutôt que des définitions nominales. Dans l'Éthique à Nicomaque, en revanche, Aristote se laisse guider par l'usage ordinaire de la langue et par les représentations dominantes, anticipant en cela l'orientation que les «analytiques » trouvent dans le langage ordinaire. Sorabji prend comme autre exemple le fait qu'Aristote néglige la distinction entre nécessité logique et nécessité extra-logique : comme ce qui lui importe la plupart du temps dans les œuvres scientifiques est la nécessité objective, une différenciation précise ne l'intéresse pas beaucoup. Il est vrai que l'Éthique à Nicomaque n'échappe pas entièrement à ces questions: par exemple, la nécessité, dans le cas du syllogisme pratique, doit-elle être comprise comme logique ou objective ? Cependant les singularités de la philosophie aristotélicienne, qui sont dues à ses intérêts scientifiques, y sont beaucoup moins prononcées, de sorte que cette œuvre correspond beaucoup mieux à l'orientation à l'époque privilégiée vers les questions logico-linguistiques. Depuis le diagnostic de Sorabji, plus de quarante ans ont passé. Il vaudrait donc la peine de rechercher quelles sont les œuvres d'Aristote aujourd'hui privilégiées par les philosophes d'orientation analytique, et surtout de se demander quelles sont les raisons de cette préférence. Car le temps et l'esprit du temps ne s'arrêtent pas non plus au seuil de la philosophie analytique, même si ce fait n'est pas volontiers reconnu par beaucoup de ses représentants ${ }^{29}$.

Traduit de l'allemand par André Laks et Joëlle Masson

27. Sorabji 1969.

28. Par exemple la définition de l'éclipse de lune, du tonnerre, ou de la quadrature du cercle.

29. Pour l'attitude des philosophes analytiques à l'égard de l'histoire, voir Sluga 1998. 


\section{BIBLIOGRAPHIE}

ACKRILL, J.L. 1955 : « $\Upsilon \Upsilon \Pi \Lambda \mathrm{OKH} \mathrm{EI} \Delta \Omega \mathrm{N} \gg$, Bulletin of the Institute of Classical Studies, 2 (1955), p. 31-35.

- : «In Defense of Platonic Division », dans O.P. Wood \& G. Pitcher (éd.), Ryle: a Collection of Critical Essays, Garden City, N. Y., 1970 (Modern Studies in Philosophy), p. 373-392.

ALLEN, R.E. (éd.) 1965 : Studies in Plato's Metaphysics, Londres-New York, 1965.

BosTOCK, D. 1994 : « Plato on Understanding Language », dans S. Everson (éd.), Language, Cambridge, 1994 (Companions to Ancient Thought, 3), p. 10-27.

CoHen, S.M. 1971 : « The Logic of The Third Man », Philosophical Review, 80 (1971), p. 448-475.

CORNFORD, F.M. 1935 : Plato's Theory of Knowledge : the Theaetetus and the Sophist of Plato, translated with a running commentary, Londres, 1935.

FreIs, R. (éd.) 1969 : The Progress of Plato's Progress, Berkeley, Cal., 1969 (Agôn. Suppl. 2).

GEACH, P. 1956 : « The Third Man Again », Philosophical Review, 65 (1956), p. 72-82.

HÄGLER, R.-P. 1983 : Platons 'Parmenides': Probleme der Interpretation, Berlin, 1983 (Quellen und Studien zur Philosophie, 18).

KroHS, U. 1998 : « Platons Dialektik im Sophistes vor dem Hintergrund des Parmenides », Zeitschrift für philosophische Forschung, 52 (1998), p. 237-256.

Lienemann, B. 2010 : Die Argumente des Dritten Menschen in Platons Dialog Parmenides: Rekonstruktion und Kritik aus analytischer Perspektive, Göttingen, 2010 (Hypomnemata, 184).

MeINWALD, C.C. 1991 : Plato's Parmenides, New York-Oxford, 1991.

- 1992: « Good Bye to the Third Man », dans R. Kraut (éd.), The Cambridge Companion to Plato, Cambridge, 1992, p. 365-396.

MignucCI, M. 1990 : «Plato's 'Third Man' Arguments in the Parmenides », Archiv für Geschichte der Philosophie, 72 (1990), p. 143-181.

QUINE W. van O. 1987: Quiddities: An Intermittently Philosophical Dictionary, Cambridge, Mass., 1987. [Trad. fr. par D. Goy-Blanquet \& T. Marchaisse, Quiddités. Dictionnaire philosophique par intermittence, Paris, 1992 (L'ordre philosophique).]

RYLE, G. 1966 : Plato's Progress, Cambridge, 1966.

SCHOFIELD, M. 1990 : «Editor's Notes », Phronesis, 35 (1990), p. 327-334

Sellars, W. 1955: «Vlastos and 'The Third Man'», Philosophical Review, 64 (1955), p. $405-437$.

- 1967: «Vlastos and 'The Third Man': a Rejoinder », dans Id., Philosophical Perspectives, Springfield, Ill., 1967, p. 55-72.

SKeltoN, A. 2010 : «William David Ross », Stanford Encyclopedia of Philosophy, August 12, 2010 [sur le site internet: http://plato.stanford.edu/entries/william-davidross/

SLugA, H. 1998 : « What Has History to Do with Me ? Wittgenstein and Analytic Philosophy », Inquiry, 41 (1998), p. 99-121.

SORABJI, R. 1969 : « Aristotle and Oxford Philosophy », American Philosophical Quarterly, 6 (1969), p. 127-135.

Teloh, H.A. \& D.J. LouZeCKy 1972 : « Plato's Third Man Argument », Phronesis, 17 (1972), p. 80-94.

Vlastos, G. 1954: «The Third Man Argument in the Parmenides », Philosophical Review, 63 (1954), p. 319-349 = D.W. Graham (éd.), G. Vlastos, Studies in Greek 
Philosophy, vol. II : Socrates, Plato and Their Tradition, Princeton, NJ, 1995, p. 166190.

- 1955: «Addenda to the Third Man Argument: A Reply to Professor Sellars », Philosophical Review, 64 (1955), p. 438-448 = D.W. Graham (éd.), G. Vlastos, Studies in Greek Philosophy, vol. II : Socrates, Plato and Their Tradition, Princeton, NJ, 1995, p. 194-203.

- 1973: « Plato’s ‘Third Man’ Argument (Parm. 132a1-b2) : Text and Logic », dans Id., Platonic Studies, Princeton, N.J., 1973, p. 342-365. 